\title{
An Algorithm of Vocabulary Enhanced Intelligent Question Answering Based on FLAT $^{1}$
}

\author{
Jing Sheng LEI ${ }^{\mathrm{a}}$, Shi Chao YE ${ }^{\mathrm{a}}$, Sheng Ying YANG ${ }^{\mathrm{a}, 2}$, Wei SONG ${ }^{\mathrm{a}}$ and \\ Guan Mian LIANG ${ }^{\mathrm{b}}$ \\ ${ }^{a}$ Department of School of Information and Electronic Engineering, Zhejiang University \\ of Science and Technology, Hangzhou, PR China \\ ${ }^{\mathrm{b}}$ Cancer Hospital of the University of Chinese Academy of Science, Hangzhou, PR \\ China
}

\begin{abstract}
The main purpose of the intelligent question answering system based on the knowledge graph is to accurately match the natural language question and the triple information in the knowledge graph. Among them, the entity recognition part is one of the key points. The wrong entity recognition result will cause the error to be done propagated, resulting in the ultimate failure to get the correct answer. In recent years, the lexical enhancement structure of word nodes combined with word nodes has been proved to be an effective method for Chinese named entity recognition. In order to solve the above problems, this paper proposes a vocabulary-enhanced entity recognition algorithm (KGFLAT) based on FLAT for intelligent question answering system. This method uses a new dictionary that combines the entity information of the knowledge graph, and only uses layer normalization for the removal of residual connection for the shallower network model. The system uses data provided by the NLPCC 2018 Task7 KBQA task for evaluation. The experimental results show that this method can effectively solve the entity recognition task in the intelligent question answering system and achieve the improvement of the FLAT model, and the average $\mathrm{F} 1$ value is 94.72
\end{abstract}

Keywords. Intelligent question and answer,Knowledge Graph,Entity recognition,Vocabulary enhancement, Residual connection

\section{Introduction}

With the continuous growth of data on the Internet, a large-scale knowledge base was born, including Freebase [1] and NLPCC KBQA. These promote the development of knowledge base question answering system (Question Answering over Knowledge Base,KBQA), which can help people naturally retrieve answers from large-scale semi-structured knowledge base. Entity recognition and attribute mapping are the main components of a ques-

\footnotetext{
${ }^{1}$ This work is supported by Natural Science Foundation of China (No. 61672337, 61972357), Zhejiang Key R\&D Program (No. 2019C03135), Basic Public Welfare Research Project of Zhejiang(LGF19F020003).

${ }^{2}$ Corresponding author: Shengying Yang, Department of School of Information and Electronic Engineering, Zhejiang University of Science and Technology, Hangzhou, 310023, PR China; E-mail: syyang@ zust.edu.cn.
} 
tion answering system based on large-scale knowledge base. Because there are many different ways to ask the same question, the deep understanding of the input question is the key to realize an intelligent question answering system based on a knowledge base. The intelligent question answering system of knowledge graph based on information extraction method can effectively locate the key entities in the question and match the correct answer from the knowledge graph.

In May 2012, Google proposed the concept of Knowledge Graph [2], which is a new type of data representation. The knowledge graph model can understand the relationship between objective things by storing entities, relationships between entities and attributes. The basic unit is a triplet, such as "entity-attribute-attribute value" or "entity-relationentity". Therefore, the knowledge graph is a high-quality corpus, which can efficiently organize and manage data information.

The knowledge graph intelligent question answering system based on the information extraction method first needs to obtain the entity information in the question sentence through the named entity recognition technology. If the entity-related information is queried in the knowledge graph, we can obtain the subgraph of the knowledge graph centered on the entity node. The candidate answer is the node or edge in the subgraph of the knowledge graph. Then, the question sentence is transformed into a question feature vector through information extraction technology. Finally, the problem feature vector and candidate answers are filtered through the classifier to find the correct answer. HUANG [3] proposed a named entity model based on LSTM and CRF, which are widely used in the entity recognition part of intelligent question answering systems based on knowledge graphs. However, this method can only be based on characters or vocabulary in Chinese, and cannot solve the Chinese entity boundary problem well.

In this article, our contributions are as follows:

1. The KBQA framework based on information extraction is proposed, and the FlatLattice model is applied to the entity recognition part of the knowledge graph intelligent question answering system.

2. Designed a vocabulary enhancement method based on FLAT model and combined with knowledge graph entities. This method can effectively solve the problem that the intelligent question answering entity recognizes the boundary of some Chinese entities. While based on characters, the vocabulary information of entities in the knowledge graph is introduced, which enriches the high-level features of the text information more specifically, thereby improving the recognition of question entities in the Chinese intelligent question answering system based on the knowledge graph.

3. By designing the entity tagging algorithm of question-and-answer pairs based on remote supervision, the data set of NLPCC2018-KBQA is labeled, so that it can be used for named entity evaluation.

4. A large number of experiments were conducted on the NLPCC 2018 Task7 KBQA task, and our model achieved good accuracy, recall rate and f1 score.

\section{Related Work}

The method is based on information extraction first needs to obtain the entity information in the question through the named entity recognition technology. If the entity-related information is queried in the knowledge graph, we can obtain the subgraph of the knowl- 
edge graph centered on the entity node. The candidate answer is the node or edge in the subgraph of the knowledge graph. Then, the question sentence is transformed into a question feature vector through information extraction technology. Finally, the problem feature vector and candidate answers is filtered through the classifier to find the correct answer.

The deep neural network model has become a research trend in named entity recognition tasks because it does not require manual feature engineering and expert knowledge in related fields [4]. Liu [5] proposed a sequence tagging framework LM-LSTM-CRF. In addition to the word-level knowledge contained in pre-trained word embeddings, this framework also integrates a character-aware neural language model, so the language model can be effectively used Extract character-level knowledge from a self-contained sequence information. Yan [6] proposed a NER architecture-TENER that uses transformer encoders to simulate character-level features and word-level features. It greatly improves performance by introducing orientation perception, distance perception and scale-free attention. Gui [7] proposed a Chinese NER model LRCNN based on convolutional neural network. This method combines vocabulary and reflection mechanism to make parallel modeling of all characters and potential words in a sentence.

BERT [8] is a language representation model based on a pre-trained deep bidirectional Transformer [9] structure proposed by Google in October 2018. Use the context information of the word to do feature extraction, dynamically adjust the word vector according to the different context information, and solve the problem of word2vec[10]. To use the pre-trained BERT, you only need to load the pre-trained model as the word embedding layer of your current task, and then build the subsequent model structure for the specific task.

According to the language characteristics of English, identifying the categories of entities is the main task of English named entity recognition task. Compared with English, Chinese has no linguistic characteristics that can determine the boundaries of entities, such as the spaces between words and words and the obvious capital letters of entity words. Therefore, determining the entity boundary is one of the research focuses of Chinese named entity task. Character-based entity recognition and word-based entity recognition after word segmentation are two common ways of Chinese named entity recognition at present. However, the semantic meaning of a sentence in Chinese will change due to different results of word segmentation, resulting in errors. Therefore, the characterbased method is generally better than the vocabulary-based method in Chinese named entity recognition. Recent studies have shown that lexical boundaries are beneficial to the determination of entity boundaries in Chinese named entity tasks. Therefore, on the basis of the character-based method, the use of vocabulary information, through the way of vocabulary enhancement, so as to improve the performance of the Chinese named entity recognition system. Zhang [11] applied lexical enhancement method to Chinese named entity recognition for the first time, and proposed a lattice structure LSTM model-Lattice LSTM for Chinese NER. The model encodes the input character sequence and all the potential words matched with the dictionary, and fuses the vocabulary information into the original cyclic neural network model. Gui [12] proposed a global semantic graph neural network (LGN), based on dictionaries, in which the graph structure is composed of characters as nodes and matched lexical information as edges. The network uses dictionary knowledge to connect the characters to obtain local combinations, while global relay nodes can capture global sentence semantics and long-range dependencies, so that 
the model of rnn is easily affected by word ambiguity. Liu [13] proposed the WC-LSTM model, which adds word information to the beginning or end of a word, which reduces the impact of word segmentation errors on the acquisition of word boundary information. At the same time, through four different strategies, the lexical information are encoded into a fixed size representation, so that effective parallelization training can be carried out. Ma [14] introduce lexical information in the embedding layer through the Soft-lexicon method. For different sequence frameworks, this method avoids the complex design of the sequence modeling architecture, and can introduce dictionary information by finetuning the character representation layer. Li [15] proposed FLAT, which transforms the grid structure into a plane structure composed of spans. Each span corresponds to a character or latent word and its position in the original lattice. At the same time, according to the fully connected self-attention mechanism in Transformer, it can well capture the long-distance dependence and use it to introduce the position vector to maintain the position information. Thus it can be seen that it is of great research significance to apply vocabulary enhancement technology to the entity recognition steps in the intelligent question answering system of Chinese knowledge graph.

\section{Methodology}

The system is mainly composed of two parts: named entity recognition and attribute mapping. Among them, the purpose of the entity recognition step is to find the entity information in the question, and the purpose of the attribute mapping step is to determine the related attributes of the question after finding the question entity. This paper will introduce the model of named entity recognition step in detail.

\subsection{Named Entity Recognition}

The entity recognition part is mainly to find the entity information in the question, which is used for the subsequent steps of attribute mapping in the intelligent question and answer. The model framework of this part of this article is shown in Figure 1. First, combine the entity vocabulary information in the knowledge graph to construct a lattice structure composed of characters and vocabulary, flatten it, and then encode the relative position of awakening according to the span of the lattice. Finally, the coding information combined with the model is used to identify the correct entity in the question.

\subsection{Dictionary Combining Entity Information Of Knowledge Graph}

This model does named entity recognition in question sentences. Except for byte points, the added word nodes is added according to the dictionary. The FLAT model uses the word segmentation information of the giga dictionary. As long as the word segmentation information appears in the question sentence, it is added as a new word node. For this task, there is more redundant information. The appearance of the entity in the question is related to but not exactly the same as the entity vocabulary in the mention2id file, such as participles, abbreviations or abbreviations. The new dictionary mainly focuses on the word segmentation, acronyms or abbreviations of the entity information in the knowledge graph through the mention2id file. The new vocabulary is more accurate, and the entity is more targeted on the vocabulary. At the same time, simplifying the vocabulary helps to improve the efficiency of the model. 


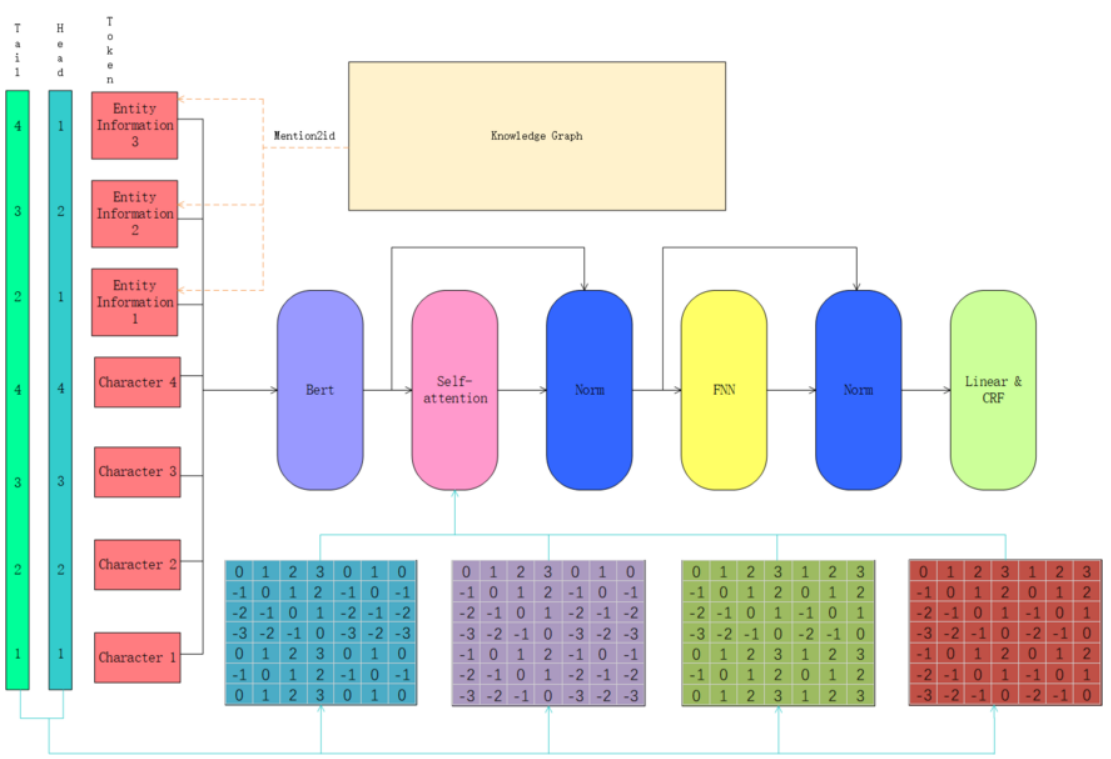

Figure 1. an algorithm of vocabulary-enhanced intelligent question-answer entity recognition based on FLAT

\subsection{Converting Lattices To FLAT Structures}

The sentence is matched with the new dictionary in the form of characters, and the obtained information is the grid. Convert it to a flat structure as shown in Figure 1. The plane grid is displayed in the form of a span. A span consists of token, head and tail. In this form, a character or vocabulary information can be marked. In order to express the position information of each token, the model uses head and tail to indicate the position index of the first and last characters of each token in the input sequence. If the token information is a single character, its head and tail are the same. Therefore, byte points are constructed by the same symbols of head and tail, and word nodes are marked by different head and tail by constructing jumping paths. And the change is recoverable, and the original structure of the plane grid can be restored.

\subsection{Relative Position Coding Of Span}

By encoding the relative position of the spans, we encode the interactions between spans of different lengths in a planar lattice structure. According to the span of head and tail, the two spans $\mathrm{Ya}$ and $\mathrm{Yb}$ of a planar lattice have three relationships: separation, intersection and inclusion. We use the continuous transformation calculation of head and tail information, and use the form of a dense vector to model their relationship. This method not only represents the relationship between the two markers, but also contains information such as the distance between words. The four relative distances of $\mathrm{Ya}$ and $\mathrm{Yb}$ are calculated by the following formula, they can represent the relationship between the two, where $h[a]$ and $t[a]$ represent the head and tail information about the span Ya, and $l_{a b}^{(h h)}$ represents The distance between the head of Ya and the tail of $\mathrm{Yb}$, the rest of $l_{a b}^{(h t)}$, $l_{a b}^{(t h)}$ and $l_{a b}^{(t t)}$ can be deduced by analogy. 


$$
\begin{aligned}
& l_{a b}^{(h h)}=h[a]-h[b] \\
& l_{a b}^{(h t)}=h[a]-t[b] \\
& l_{a b}^{(t h)}=t[a]-h[b] \\
& l_{a b}^{(t t)}=t[a]-t[b]
\end{aligned}
$$

As shown in the following formula, referring to the method of Vaswani et al. [9], and through the nonlinear transformation of the above four distance formulas, the final relative position coding of the span is obtained.

$$
\begin{aligned}
& P_{d}^{(2 k)}=\sin \left(d / 10000^{2 k / d_{m} \text { odel }}\right) \\
& P_{d}^{(2 k+1)}=\cos \left(d / 10000^{2 k / m o d e l}\right) \\
& M_{a b}=\operatorname{ReLU}\left(W_{m}\left(P_{l_{a b}^{(h h)}} \oplus P_{l_{a b}^{(h t)}} \oplus P_{l_{a b}^{(t h)}} \oplus P_{l_{a b}^{(t t)}}\right)\right.
\end{aligned}
$$

Where $\mathrm{d}$ is $l_{a b}^{(h h)}, l_{a b}^{(h t)}, l_{a b}^{(t h)}, l_{a b}^{(t t)}$, Wm is a learnable parameter, and $\mathrm{k}$ represents the position-coded dimension index. Then, according to the method of Dai et al. [16], a variant of self-attention is used to encode the relative span position, and the calculation formula is as follows:

$$
A_{a, b}^{*}=W_{q}^{\mathrm{T}} E_{Y_{a}}^{\mathrm{T}} E_{Y_{b}} W_{k, E}+W_{q}^{\mathrm{T}} E_{Y_{a}}^{\mathrm{T}} M_{a b} W_{k, M}+u^{\mathrm{T}} E_{Y_{b}} W_{k, E}+v^{\mathrm{T}} M_{a b} W_{k, M}
$$

Among them, $W_{q}, W_{k, M}, W_{k, E} \in \mathbb{R}^{l_{\text {model }} \times l_{\text {head }}}$ and $\mathrm{u}, \mathrm{v} \in \mathbb{R}^{l_{\text {head }}}$ are the learnable parameters.

\subsection{Remove Residual Connection}

The purpose of residual connection is to solve the degradation problem that when the layers of the training network are getting deeper and deeper, the training effect is not as good as the relatively shallow network. By adding shortcut connections, the residual network makes the training model easier to optimize. The residual block consists of a multi-layer network containing shortcut connections. A residual block is represented by the following formula, and the residual network is composed of residual blocks.

$$
X_{l+1}=X_{1}+F\left(x_{1}, W_{1}\right)
$$

The additional $\mathrm{X}$ term can solve the problem of gradient disappearance in the process of back propagation. Because when the network calculates the partial derivative of $\mathrm{X}$, there is an extra constant 1.

Transform uses the structure of a six-layer encoder-decoder, which consists of a selfattention mechanism and a feed-forward network (FFN), with residual connections and layer normalization added after each sublayer. The calculation formula is as following: 


$$
\begin{aligned}
& \operatorname{Att}(A, V)=\operatorname{softmax}(A) V \\
& A_{a b}=\frac{Q_{i} K_{j}^{T}}{\sqrt{d_{\text {head }}}} \\
& {[Q, K, V]=E_{x}\left[W_{q}, W_{k}, W_{v}\right]}
\end{aligned}
$$

Among them, $W_{q}, W_{k}, W_{v} \in \mathbb{R}^{l_{\text {model }} \times l_{\text {head }}}$ are the learnable parameters, and the model in this paper replaces $A_{a b}$ with $A_{a, b}^{*}$ in formula 8.FLAT inherited this structure directly, but he actually only used one layer of encoder. We think this is a shallower network compared to transform, so the residual connection is removed and only layer normalization is used. Finally, the character representation is put into the output layer, and then the entity recognition task is completed through the Conditional Random Field (CRF) [17].

\section{Experiments}

\subsection{Experimental Data And Preprocessing}

This experiment uses data provided by the NLPCC 2018 Task7 KBQA task. The task data contains 24,479 one-to-one corresponding question and answer data pairs and the required knowledge graph. The knowledge graph provided by this data contains $6,502,738$ entities, 587,875 attributes, and 43,063,769 triples. It is currently one of the large-scale open domain knowledge graphs in Chinese. Each row in the knowledge graph stores a triple (entity, attribute, attribute value/entity).

Because the NLPCC2018-KBQA dataset is not a dataset in the task area of named entity recognition, the 24479 question-and-answer pairs of data it provides do not clearly indicate the location of the entity. Therefore, this experiment is based on the idea of remote supervision. According to the triples of the provided knowledge graph, remote supervision annotates the entity sequence of the question in the question and answer pair. In this way, a data set with entity sequence annotations that can be used in the entity recognition step is obtained.

The key idea of the question-and-answer entity labeling algorithm based on remote supervision is to deduce the corresponding triples of each question-and-answer pair from the triplet knowledge base. For the knowledge graph triplet set, when there is a triple (e, $\mathrm{r}, \mathrm{s}), \mathrm{s}$ is included in the answer of a certain question and answer pair, and $\mathrm{e}$ is included in the corresponding question, then the question is considered question The sentence entity matches successfully. Find from the question whether the entity in the corresponding triple is included. If the entity is not included, use the mention2id file to replace the entity name with its corresponding synonym to continue the search. After finding the entity, it is written in the NER training corpus format, and each word is given a corresponding label such as B-ENT, as shown in Figure 2.

The collection of 24479 questions and answer data pairs, mention2id files and 43063796 knowledge graph triples provided by the NLPCC 2018 Task7 KBQA task are preprocessed through 2.1.2 steps. First, a mapping dictionary AnswerDict with a length of 21269 is formed, that is, the size of the dictionary key when the question and answer 


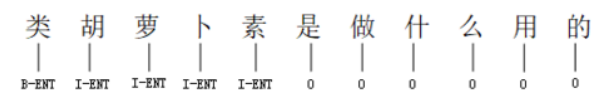

Figure 2. training expectation format after labeling

Table 1. KGFLAT model parameter list

\begin{tabular}{cc}
\hline batch & 8 \\
learning rate & 0.0005 \\
optimizer & SGD \\
-momentum & 0.9 \\
head & 8 \\
head_dim & 16 \\
output dropout & 0.3 \\
warmup & 0.1 \\
\hline
\end{tabular}

pair is built, which is the number of answers that are not repeated in the data. Find from the question whether it contains the entity in the corresponding triple. If it does not contain the entity, replace the entity name with its corresponding synonym through the mention2id file to continue the search. Even then, there are still entities that cannot be found in the corresponding question. So, discard this part of the data. Finally, 15640 valid questions and answers are labeled successfully by the question-and-answer entity labeling algorithm based on remote supervision.

Split the dataset $80 \%$ as the training set, $10 \%$ as the validation set, and $10 \%$ as the test set. The number of training sets, verification sets and test sets are 12512, 1564 and 1564 respectively.At the same time, in order to obtain a reliable and stable model, the ten-fold cross-validation (10-fold cross-validation) method [18] is used for training. Models that work well on the validation set are evaluated on the test set. According to the common evaluation criteria of intelligent question and answer, the accuracy, recall rate and average $\mathrm{F} 1$ value were used to evaluate the experimental results.

\subsection{Experimental Settings And Parameter Optimization}

The experiment was carried out in the Ubuntu 18.04 system, where the CPU was an Intel i5-7500 processor with 4 cores, the GPU was NVIDIA TITAN XP-12GB, and the memory was $16 \mathrm{~GB}$. The model training process is carried out on the GPU, and the experimental code adopts the Pytorch deep learning framework.

The dimension of the input word vector in the model is 50, the Batch Size is set to 8, the learning rate is set to 0.0005 , and the parameter optimizer uses the SGD optimizer. More model parameters are shown in Table 1:

\subsection{Experimental Results And Analysis}

In order to verify the advancement of this model, the classical models BI-LSTM[3], Lattice LSTM [11], TENER [6], LRCNN [7], FLAT [15] and BERT-LSTM are tested on this dataset. The experimental results are shown in table 2.

Comparing the classic model and the current Chinese named entity recognition model with better performance, as shown in Table 2, the model proposed in this paper has 
Table 2. Comparison of KGFLAT with the classic model on the NLPCC 2018 Task7 KBQA task data set

\begin{tabular}{cccc}
\hline Model & $\mathrm{P}$ & $\mathrm{R}$ & $\mathrm{F} 1$ \\
\hline LSTM+CRF & 88.00 & 86.88 & 87.44 \\
Lattice LSTM & 89.76 & 89.82 & 89.79 \\
TENER & 90.50 & 90.27 & 90.38 \\
LRCNN & 91.32 & 90.91 & 91.11 \\
FLAT & 91.99 & 91.99 & 91.99 \\
BERT+LSTM+CRF & 93.22 & 92.64 & 92.93 \\
BERT +FLAT & 93.92 & 93.70 & 93.81 \\
BERT+FLATND & 94.50 & 94.56 & 94.52 \\
BERT+FLATNR & 94.29 & 94.47 & 94.38 \\
BERT+KGFLAT & 94.75 & 94.69 & 94.72 \\
\hline
\end{tabular}

achieved better results. As shown in Table 2, compared with the classic models based on recurrent neural networks and combined with Lattice lattice structure, such as Bi-LSTM, LatticeLSTM and BERT-LSTM, the average F1 value of the model in this paper increased by $7.28,4.93$, and 1.79 percentage points, respectively; compared with the model TENER, LRCNN and FLAT increased by $4.34,3.61$, and 2.73 percentage points respectively. The performance of this model in this data is significantly improved, which is better than the performance of the above model in this data.

The FLAT model uses a character vector and word vector which are trained by Giga Dictionary. In this paper, the pre-training model Bert is used to train the embedding. On this basis, the performance of the model FLATND after modifying the dictionary combined with the knowledge graph entity information and the model FLATNR after de-residual connection on the data is the same as the performance of the original FLAT model on the data Promote. KGFLAT not only uses the new dictionary but also removes the residual connections. Compared with the original FLAT model, the average F1 value of this model is improved by $0.91 \%$. The model in this paper verifies the effectiveness of the new dictionary and de-residual connection to improve the ability of the FLAT model, and the average F1 value reaches 94.72 .

\section{Conclusion}

Entity recognition is a difficulty in building an intelligent question answering system based on knowledge graph. The model proposed in this paper solves this problem well. Traditional entity recognition algorithms is mainly based solely on character information or word information. If it is a character -based approach, words are often rarely considered, so a large amount of information contained in the vocabulary can not be used. Lexical boundaries usually play an important role in entity boundaries, and the entity information in the knowledge graph can make entity recognition algorithms more targeted. Based on the knowledge corpus of NLPCC2018-KBQA, this paper proposes and applies the question-answer entity labeling algorithm based on remote supervision and the wordenhanced entity recognition algorithm based on FLAT. The above data and algorithms is used to build an intelligent question answering system. In the entity recognition step, 
the model proposed in this paper uses vocabulary enhancement technology, based on the FLAT model and through the creation of a new dictionary and de-residual connection to achieve the promotion of the FLAT model.

\section{References}

[1] Kurt Bollacker, Colin Evans, Praveen Paritosh, Tim Sturge, and Jamie Taylor. Freebase: a collaboratively created graph database for structuring human knowledge. In Proceedings of the 2008 ACM SIGMOD international conference on Management of data, pages 1247-1250, 2008.

[2] Thomas Steiner, Ruben Verborgh, Raphaël Troncy, Joaquim Gabarro, and Rik Van de Walle. Adding realtime coverage to the google knowledge graph. In 11th International Semantic Web Conference (ISWC 2012). Citeseer, 2012.

[3] Zhiheng Huang, Wei Xu, and Kai Yu. Bidirectional lstm-crf models for sequence tagging. arXiv preprint arXiv: 1508.01991, 2015.

[4] Joey Tianyi Zhou, Hao Zhang, Di Jin, Xi Peng, Yang Xiao, and Zhiguo Cao. Roseq: Robust sequence labeling. IEEE transactions on neural networks and learning systems, 31(7):2304-2314, 2019.

[5] Liyuan Liu, Jingbo Shang, Xiang Ren, Frank Xu, Huan Gui, Jian Peng, and Jiawei Han. Empower sequence labeling with task-aware neural language model. In Proceedings of the AAAI Conference on Artificial Intelligence, volume 32, 2018.

[6] Hang Yan, Bocao Deng, Xiaonan Li, and Xipeng Qiu. Tener: Adapting transformer encoder for named entity recognition. arXiv preprint arXiv:1911.04474, 2019.

[7] Tao Gui, Ruotian Ma, Qi Zhang, Lujun Zhao, Yu-Gang Jiang, and Xuanjing Huang. Cnn-based chinese ner with lexicon rethinking. In IJCAI, pages 4982-4988, 2019.

[8] Jacob Devlin, Ming-Wei Chang, Kenton Lee, and Kristina Toutanova. Bert: Pre-training of deep bidirectional transformers for language understanding. arXiv preprint arXiv:1810.04805, 2018.

[9] Ashish Vaswani, Noam Shazeer, Niki Parmar, Jakob Uszkoreit, Llion Jones, Aidan N Gomez, Lukasz Kaiser, and Illia Polosukhin. Attention is all you need. arXiv preprint arXiv:1706.03762, 2017.

[10] Tomas Mikolov, Kai Chen, Greg Corrado, and Jeffrey Dean. Efficient estimation of word representations in vector space. arXiv preprint arXiv:1301.3781, 2013.

[11] Yue Zhang and Jie Yang. Chinese ner using lattice 1stm. arXiv preprint arXiv:1805.02023, 2018.

[12] Tao Gui, Yicheng Zou, Qi Zhang, Minlong Peng, Jinlan Fu, Zhongyu Wei, and Xuan-Jing Huang. A lexicon-based graph neural network for chinese ner. In Proceedings of the 2019 Conference on Empirical Methods in Natural Language Processing and the 9th International Joint Conference on Natural Language Processing (EMNLP-IJCNLP), pages 1039-1049, 2019.

[13] Wei Liu, Tongge Xu, Qinghua Xu, Jiayu Song, and Yueran Zu. An encoding strategy based wordcharacter 1stm for chinese ner. In Proceedings of the 2019 Conference of the North American Chapter of the Association for Computational Linguistics: Human Language Technologies, Volume 1 (Long and Short Papers), pages 2379-2389, 2019.

[14] Ruotian Ma, Minlong Peng, Qi Zhang, and Xuanjing Huang. Simplify the usage of lexicon in chinese ner. arXiv preprint arXiv: 1908.05969, 2019.

[15] Xiaonan Li, Hang Yan, Xipeng Qiu, and Xuanjing Huang. Flat: Chinese ner using flat-lattice transformer. arXiv preprint arXiv:2004.11795, 2020.

[16] Zihang Dai, Zhilin Yang, Yiming Yang, Jaime Carbonell, Quoc V Le, and Ruslan Salakhutdinov. Transformer-xl: Attentive language models beyond a fixed-length context. arXiv preprint arXiv:1901.02860, 2019.

[17] John Lafferty, Andrew McCallum, and Fernando CN Pereira. Conditional random fields: Probabilistic models for segmenting and labeling sequence data. 2001.

[18] Peng Chen, HK Feng, CC Li, GJ Yang, JS Yang, WP Yang, and SB Liu. Estimation of chlorophyll content in potato using fusion of texture and spectral features derived from uav multispectral image. Transactions of the Chinese Society of Agricultural Engineering, 35(11):63-74, 2019. 